\title{
Hanseníase e migração: correlação espacial em um Estado hiperendêmico da
}

\section{Amazônia brasileira}

\author{
Leprosy and migration: spatial correlation in a hyperendemic State in the brazilian Amazon \\ Lepra y migración: correlación espacial en un Estado hiperendémico de la Amazonía brasileña
}

Waltair Maria Martins Pereira

ORCID: https://orcid.org/0000-0002-7383-0318

Universidade Federal do Pará, Brasil

E-mail: vweapaz@gmail.com

Rebeca Lima Braga

ORCID: https://orcid.org/0000-0003-1955-1315

Universidade Federal do Pará, Brasil

E-mail: rebeca.braga @ics.ufpa.br

Emanuele Rocha da Silva

ORCID: https://orcid.org/ 0000-0002-9109-4158

Universidade Federal do Pará, Brasil

E-mail: emanueleersilva@gmail.com

José Natanael Gama dos Santos

ORCID: https://orcid.org/0000-0001-7298-9602

Universidade Federal do Pará, Brasil

E-mail: josenatanelsantos@hotmail.com

Belmiro Figueiredo Vinente Neto

ORCID: https://orcid.org/0000-0003-1827-6964

Universidade Federal do Pará, Brasil

E-mail: bnvinente@gmail.com

Joao Victor Filgueiras Mota

ORCID: https://orcid.org/0000-0002-6346-684X

Universidade Federal do Pará, Brasil

E-mail: joao.mota@ics.ufpa.br

Raiula Gabriela da Silva Teixeira

ORCID: https://orcid.org/0000-0002-5779-7968

Universidade Federal do Pará, Brasil

raiulagabriela@gmail.com

Ivone Diniz Chaquiam

ORCID: https://orcid.org/0000-0003-2287-326X

Universidade Federal do Pará, Brasil ichaquiam@gmail.com

Erika Martins Pereira

ORCID: https://orcid.org/0000-0002-2247-0017

Universidade Federal do Maranhão

E-mail: erika.mp@ufma.br

Igor da Silva Narvaes

ORCID: https://orcid.org/0000-0002-9950-895X

Instituto Nacional de Pesquisas Espaciais, Brasil

E-mail: igornarvaes@gmail.com

Marcos Adami

ORCID: https://orcid.org/0000-0003-4247-4477 Instituto Nacional de Pesquisas Espaciais, Brasil

E-mail: marcos.adami@inpe.br

Lourdes Maria Garcez

ORCID: https://orcid.org/0000-0003-2231-3561 Instituto Evandro Chagas, Brasil E-mail: luma.garcez@gmail.com

\section{Resumo}

Este estudo teve como objetivo identificar a dependência espacial das taxas de detecção de hanseníase na população geral e em menores de 15 anos com a proporção de migração, no estado do Pará. Foi realizada pesquisa com desenho de estudo ecológico em uma série histórica de casos de novos de hanseníase, entre os anos de 2006 a 2015, a partir de do banco de dados do Sistema de Informação de Agravos Notificáveis do Ministério da Saúde, referentes ao Estado. A evolução temporal da taxa de detecção geral, padronizada pela idade, apresentada geograficamente, identificou um corredor de muito hiperendemicidade, no sentido Sudoeste-Sudeste do Estado e dois aglomerados de municípios, também de muito hiperendemicidade, na fronteira com os estados do Tocantins e do Maranhão. A análise espacial 
demonstrou que as taxas médias na população em geral e em menores de 15 anos e a proporção de migração apresentaram autocorrelação espacial positiva, permitindo identificar clusters localizados nas áreas Sudeste, Sudoeste, Centro Norte e Nordeste do Estado. Nessas áreas estão concentrados os projetos de exploração mineral e de agropecuária e foram construídas duas Usinas Hidroelétricas nas décadas de 1960 e 2000, sendo favorecidas por rede viária e ferroviária consolidada que é usada para o escoamento dos produtos e para o intenso fluxo migratório das pessoas. Esta pesquisa evidencia que a hanseníase apresenta alta magnitude com característica de transmissão e que os 2/3 da área territorial que expressam hiperendemicidade, a destacando como sério problema de saúde pública no estado do Pará.

Palavras-chave: Hanseníase; Aplicações da epidemiologia; Análise espacial; Doença negligenciada; Dinâmica populacional.

\begin{abstract}
This study aimed to identify the spatial dependence on leprosy detection rates in the general population and in children under 15 years of age with the migration proportion, in Pará state. The research was carried out with an ecological study design in a historical series of leprosy new cases, between 2006 and 2015, from the Ministry of Health's Notifiable Diseases Information System database, referring to the State. The temporal evolution of the general detection rate, standardized by age, presented geographically, identified a corridor of great hyperendemicity of the disease, in the Southwest-Southeast of the State and two municipalities clusters, also of very hyperendemicity, on the border with Tocantins and Maranhão States. The spatial analysis showed that the average rates in the general population and under 15 years of age and the proportion of migration showed positive spatial autocorrelation, allowing to identify clusters located in the Southeast, Southwest, North, and Northeast areas of the State. Mineral exploration and agriculture projects are concentrated in these areas, where two Hydroelectric Power Plants were built in the 1960s and 2000s, favored by a consolidated road and rail network that is used for the flow of products and for the intense people migratory flow. This research shows that leprosy has a high magnitude with active transmission characteristics and that $2 / 3$ of the territorial area expresses hyperendemicity, highlighting it as a serious public health problem in Pará state.
\end{abstract}

Keywords: Leprosy; Uses of epidemiology; Spatial analysis; Neglected diseases; Population dynamics.

\begin{abstract}
Resumen
Este estudio tuvo como objetivo identificar la dependencia espacial de las tasas de detección de lepra en la población general y en menores de 15 años con la proporción de migración, en el estado de Pará. La investigación se realizó con un diseño de estudio ecológico en una serie histórica de casos de lepra nueva, entre los años 2006 a 2015 , de la base de datos del Sistema de Información de Enfermedades Notificables del Ministerio de Salud, referido al Estado. La evolución temporal de la tasa de detección general, estandarizada por edad, presentada geográficamente, identificó un corredor de gran hiperendemicidad, en la dirección Suroeste-Sureste del Estado y dos conglomerados de municipios, también de muy hiperendemicidad, en el límite con los estados de Tocantins y el Maranhão. El análisis espacial mostró que las tasas promedio, en la población general y en niños menores de 15 años y la proporción de migración presentaron autocorrelación espacial positiva, permitiendo identificar conglomerados ubicados en las áreas Sudeste, Sudoeste, Norte y Nordeste del Estado. En estas áreas se concentran proyectos de exploración minera y agricultura y en las décadas de 1960 y 2000 se construyeron dos Plantas Hidroeléctricas, favorecidas por una red vial y ferroviaria consolidada que se utiliza para el flujo de productos y para el intenso flujo migratorio de personas. . Esta investigación muestra que la lepra tiene una alta magnitud con características de transmisión y que 2/3 del área territorial expresan hiperendemicidad, destacándola como un grave problema de salud pública en el estado de Pará.
\end{abstract}

Palabras clave: Lepra; Usos de la epidemiología; Análisis espacial; Enfermedades desatendidas; Dinámica poblacional.

\title{
1. Introdução
}

A hanseníase é uma doença infectocontagiosa de evolução crônica que afeta principalmente o tecido cutaneomucoso e os nervos periféricos. O agente infeccioso é o Mycobacterium leprae, que ao penetrar nas células nervosas, preserva a virulência mantendo alto poder imunogênico para o desenvolvimento de incapacidade física, que predispõe o indivíduo a grande carga de estigma, favorecendo a exclusão e dificultando as relações sociais e as atividades econômicas (Cury at al., 2012; Brasil, 2014a).

Registros da Organização Mundial da Saúde (OMS) apontam que existe uma tendência mundial da redução da detecção de casos novos e da prevalência da doença, possivelmente determinados pela introdução do tratamento poliquimioterápico (PQT) e pela adoção de políticas de controle e eliminação, que são pactuadas e repactuadas a cada 
quinquênio. Entretanto 14 países concentram a carga global da doença com $95 \%$ das notificações, e entre eles a Índia $(60,38 \%)$, o Brasil $(16,17 \%)$ e a Indonésia $(8,15 \%)$, foram responsáveis por 84,7\% do total das notificações (WHO, 2015).

O Brasil manteve, nas últimas décadas, a situação mais desfavorável na região das Américas. Embora tenha registrado decréscimo nas taxas de detecção na população em geral e em menores de 15 anos, no período de 2006 a 2015 , as Regiões Norte, Centro-Oeste e Nordeste, apresentaram as maiores endemicidade, conformando aglomerados de estados, que mantiveram a transmissão da doença e ratificaram ser um grande problema de Saúde Pública, cujo programa de eliminação recebe priorização por parte do Ministério de Saúde (Brasil, 2014b, Rodrigues et al., 2020).

A ocorrência da hanseníase é influenciada por fatores naturais como o clima, o relevo, os tipos de vegetação e determinados ecossistemas e fatores sociais, destacando-se as condições desfavoráveis representadas pelas dimensões de condições de vida como a econômica, a situação higiênico-sanitária, os fatores específicos dos indivíduos e a situação comportamental dentro das relações sociais, que definem a heterogeneidade espacial como uma das características da endemicidade da hanseníase (Brook at al., 2015; Rodrigues at al., 2020).

Como importante componente dos fatores sociais que contribuem para alterar os perfis epidemiológicos, o movimento migratório se destaca, por impactar tanto na área de evasão como na de recepção de migrantes. O desenvolvimento econômico e as urbanizações decorrentes desse movimento levam às mudanças de padrões de adoecimento e da distribuição geográfica das doenças, consequentes da circulação das pessoas, dos aglomerados constituídos e das diversas condições socioeconômicos enfrentadas no contexto do processo (Monken \& Barcellos, 2008).

Vários estudos vêm sendo realizados no Estado do Pará com o intuito de identificar as associações e as correlações que compõem os fatores determinantes do processo de adoecimento por hanseníase, entretanto identificar a correlação com a migração é uma abordagem oportuna e significativa, considerando que existem atividades de mineração no estado, com projetos situados em 40 de seus municípios. Ademais, há instaladas duas Usinas Hidroelétricas, a de Tucuruí, nas décadas de 60/70, e a de Belo Monte na década de 2000, que produzem grandes impactos demográficos e socioeconômicos em diversos municípios, principalmente da área sudeste do Estado (Da Silva, 2016; IBGE, 2010; Giraldin, 2015).

Com este trabalho, objetivou-se identificar a dependência espacial das taxas de detecção de hanseníase na população geral e em menores de 15 anos com a proporção de migração, no Estado do Pará, em uma série histórica.

\section{Metodologia}

Foi realizada uma pesquisa exploratória quantitativa com desenho de estudo ecológico, em uma série histórica de casos novos de hanseníase, no período de 2006 a 2015 (Pereira A.S. et al., 2018).

O estudo foi realizado no Estado do Pará, composto por 144 municípios que em 2015 contava com uma população de 8.104.880 habitantes, com 50,5\% (4.092.964) do gênero masculino e 49,5\% (4.011.916) do gênero feminino. A população é bem miscigenada, sendo formada por indígenas, negros, europeus, ribeirinhos e asiáticos.

Com extensão territorial de $1.247 .689,76 \mathrm{~km}^{2}$, ocupa $15 \%$ do território nacional e $45 \%$ do território da Região Norte. Está situado inteiramente na Amazônia e faz limites ao norte com o Amapá, a nordeste com o oceano Atlântico, a leste com o Maranhão, a sudeste com o Tocantins, ao sul com o Mato Grosso, a oeste com o Amazonas, a noroeste com Roraima e ao extremo norte com o Suriname e a Guiana. Apresenta clima equatorial quente e úmido; temperatura média anual de $24^{\circ} \mathrm{C}$ a $26^{\circ} \mathrm{C}$ e índice pluviométrico de $2.800 \mathrm{~mm}$ ao ano.

O Pará tem no extrativismo mineral sua principal atividade econômica (ferro, bauxita, manganês, calcário, ouro, estanho), o alumínio e o minério de ferro sendo os principais produtos de exportação. O extrativismo vegetal se mantém importante, porém, nem sempre de forma sustentável. Outras atividades, como a agricultura, a pecuária e o setor de serviços se 
destacam em muitos municípios e sedes urbanas; as indústrias e, de forma lenta, o turismo, vêm se destacando (Figura 1 e Figura 5) (Costa et al., 2011; FAPESPA, 2015).

Figura 1 - Mapa da América Latina com o Mapa do Brasil em destaque e área geográfica do estado do Pará em destaque no mapa do Brasil, 2020.

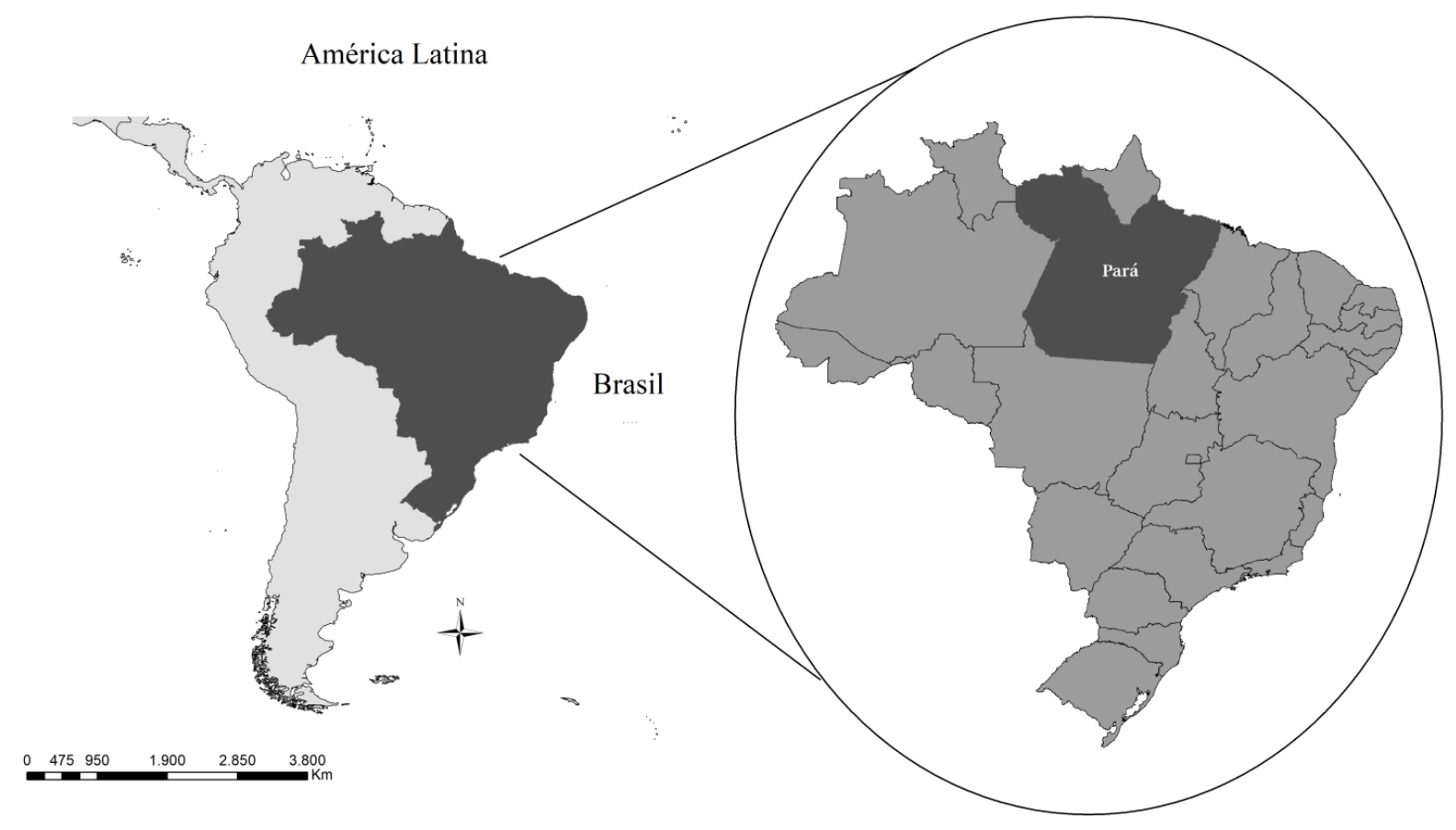

Fonte: IBGE (2015).

A população de estudo foi constituída por indivíduos que adoeceram de hanseníase, notificados e incluídos no Sistema de Informações de Agravos Notificáveis (Sinan), pelas Secretarias Municipais de Saúde, como residentes no Estado do Pará. Foram excluídos os casos notificados como hanseníase que não tinham o Estado do Pará como local de residência; casos que, após revisão técnica, tiveram que ser excluídos por equívocos no diagnóstico.

O parâmetro de avaliação para o indicador taxa de detecção geral e para menores de 15 anos foi aplicado conforme estabelecido pela OMS, e utilizado pelo MS do Brasil. Para a população em geral, área de hiperendemicidade igual e superior a 40 casos por 100 mil habitantes; muito alta endemicidade, entre 20,00 e 39,99 casos por 100 mil habitantes; alta endemicidade de 10,00 a 19,99 por 100 mil habitantes, média endemicidade de 2,00 a 9,99 casos por 100 mil habitantes. Todavia devido aos elevados valores das taxas de detecção de hanseníase no Pará, foi adicionada nova classificação, denominada "muito hiperendêmico" que engloba o valor da taxa de detecção geral superior a 100 casos/100.000 hab. (Brasil, 2016a; Monteiro et al., 2015).

Os dados demográficos foram aqueles disponibilizados em fontes secundárias de domínio público, disponíveis no banco de dados do Atlas Brasil 2013 (IPEA, 2013). Os dados cartográficos de identificação dos limites municipais foram levantados em fontes secundárias oficiais nos níveis Federal, Estadual e Municipal, de domínio público, disponíveis no banco de dados do IBGE (IBGE, 2010).

Os dados epidemiológicos dos casos notificados como hanseníase, foram disponibilizados pela Secretaria de Estado de Saúde Pública (SESPA) no formato de arquivo Data Base File ( $d b f$ ), que foi convertido para Structure Query Language (Linguagem $S Q L$ ). Através do Software Programa livre para o sistema gerenciador de banco de dados denominado MySQL, do 
também sistema gerenciador de banco de dados denominado Microsoft SQLSERVER e PostgreSQ (HeidiSQL) foi realizada a depuração dos dados, no sentido de filtrar as variáveis utilizadas para a construção das taxas de detecção de hanseníase. Para a correção de possíveis vieses e obtenção dos dados de forma tabular, utilizou-se o formato Comma-Separated Values (.csv). Posteriormente foi convertido para o formato de um arquivo com extensão eXceL Spreadsheed (.xls).

Ao banco de dados geográficos do IBGE, que contém o conjunto de dados dos municípios do Estado do Pará no formato shapefile (*.shp), de domínio público, foi realizada uma junção do banco (.xls), compondo assim um novo banco denominado de BDgeo, de onde foi oportunizado o processamento dos dados geográficos e a elaboração dos indicadores para a análise espacial.

Para a realização da análise espacial foram selecionados três indicadores, sendo um demográfico (proporção de migração) e dois epidemiológicos (taxa média de detecção de hanseníase, na população geral e em menores de 15 anos).

A identificação da autocorrelação espacial e a presença de cluster foram obtidas pela realização da análise espacial utilizando o software GeoDa for Windows (versão 1.12.1.129), software livre e de código aberto (Anselin, 2016). Foi realizada a regressão espacial para identificar a correlação das variáveis independentes (proporções de migração) com as dependentes (taxas de detecção de hanseníase na população geral e em menores de 15 anos), com o encontro das correlações de significância estatística, no nível de $\mathrm{p}$-valor $=0,05$ a p-valor $=0,001$.

Foi gerada a matriz de vizinhança a partir de uma estimativa de segunda ordem de contiguidade. Posteriormente, através de cruzamento de uma variável dependente com a independente, de forma sucessiva, foram gerados os Diagramas de Espalhamento de Moran, considerando os quatro quadrantes, a saber: quadrante superior direito com valores positivos (+/+), e médias positivas, e quadrante inferior esquerdo com valores negativos e médias negativas (-/-), que indicam pontos de associação espacial ou autocorrelação espacial positiva, no sentido de uma localização possuir vizinhos com valores semelhantes, sendo referido como autocorrelação espacial alta-alta e baixa-baixa, respectivamente. Em contraste, o quadrante inferior direito, com valores negativos, e, médias positivas (-/+), e, superior esquerdo, valores positivos, e, médias negativas (+/-), indicam pontos de associação espacial negativos, com vizinhos com valores diferentes, indicando pontos de transição entre diferentes padrões espaciais, sendo referido como autocorrelação espacial alta-baixa e baixa-alta, respectivamente (Nunes, 2013).

Posteriormente foi calculado o Índice de Moran Local ou LISA Map, que explorou o grau de dependência a partir de uma estimativa de segunda ordem de contiguidade, ou seja, a existência de covariância espacial entre os agrupamentos de municípios.

\section{Resultados}

O estado do Pará acumulou, no período de 2006 a 2015 a notificação de 38.859 casos novos de hanseníase com $69,92 \%$ ( $p<0,0001)$ dos casos residentes em áreas urbanas. Desse total 11,21\% (4.355/38.859) eram menores de 15 anos de idade. Todos os casos foram georreferenciados proporcionando a visibilidade de uma taxa média de detecção, na população em geral, de 51,35 casos para cada 100 mil habitantes.

Ao analisar as taxas médias, padronizadas, de detecção da hanseníase, na população em geral, aglutinadas para o período estudado (2006 a 2015), observou-se a formação de um corredor de muito hiperendemicidade no sentido sudoestesudeste-nordeste do Estado, composto pelos municípios de Altamira; Novo Progresso; Rurópolis; Senador José Porfírio; Anapu; Goianésia do Pará; Novo Repartimento; Jacundá; Abel Figueiredo; Dom Eliseu; Rondon do Pará e Paragominas, bem como dois importantes aglomerados (cluster), com municípios sendo o mais extenso formado pelos municípios de Xinguara; Sapucaia; Curionópolis; Eldorado dos Carajás e Paraupebas e o menos extenso pelos municípios de Conceição do Araguaia, 
Redenção e Floresta do Araguaia. Observa-se ainda o município de Mocajuba, localizado na área nordeste, isolado do corredor e dos dois aglomerados, com taxa de detecção classificada como muito hiperendemicidade (Figura 2).

Figura 2 - Distribuição geográfica da classificação de endemicidade das taxas médias padronizadas de detecção de hanseníase na população em geral, e a malha viária. Municípios do Estado do Pará, Brasil, para o período 2006 a 2015.

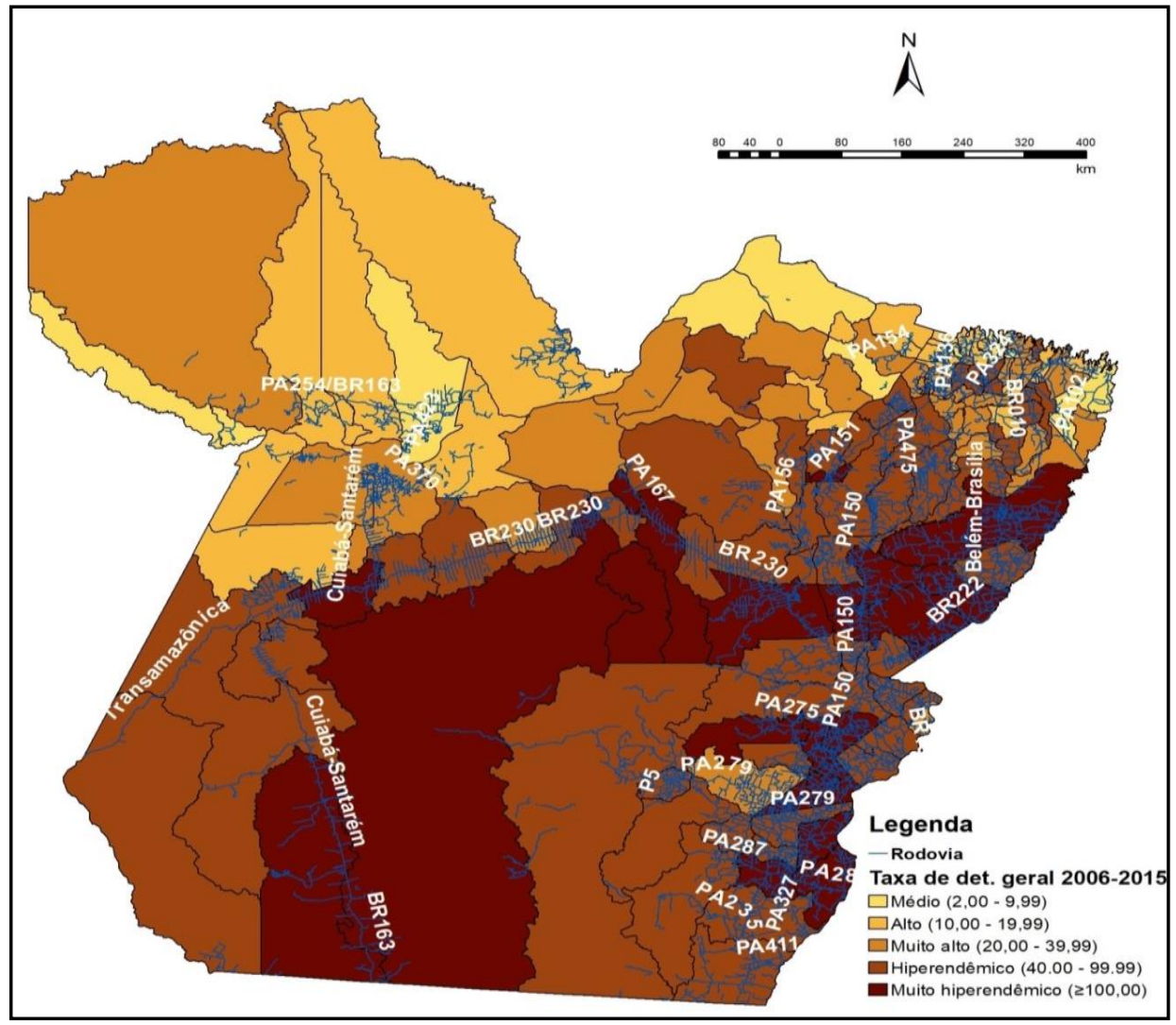

Fonte: SINAN, 2017; IBGE, 2010b; shapefile GeoDa para Windows, versão 1.12.1.129.

Observou-se que no trajeto do corredor de muito hiperendemicidade da hanseníase, encontra-se a BR-163, que tem início no Rio Grande do Sul e chega até os municípios de Santarém e Rurópolis. A BR-230, denominada de Transamazônica, corta os estados brasileiros da Paraíba, Ceará, Piauí, Maranhão, Tocantins, Pará e Amazonas, mesmo não estando totalmente pavimentada apresenta tráfego intenso. No Pará, essa rodovia, proporciona acesso os municípios de Marabá, Altamira e Itaituba. A BR-222, que atende os Estados do Ceará, Piauí, Maranhão e Pará e neste Estado atende os municípios de Marabá e Dom Elizeu, onde se liga a BR-010, denominada Belém-Brasília (IBGE, 2010) (Figura 2).

O tráfego interno no Estado é assegurado pelas rodovias, PA-150; PA- 279; PA-275; PA-287 e PA-327 importantes vias de acesso para os municípios que compõem os dois aglomerados ou cluster na área sudeste do Estado (IBGE, 2010). A rodovia PA-150, liga as áreas nordeste a sudeste do Estado, corta os municípios de Goianésia do Pará, Jacundá, Nova Ipixuna e Marabá, municípios que receberam influência do projeto da construção da usina hidroelétrica de Tucuruí. A PA- 279 liga os municípios de Água Azul do Norte, Tucumã, Ourilândia do Norte e São Feliz do Xingu, e se unindo mais adiante à BR-155, que proporciona acesso a Xinguara e ao estado de Mato Grosso (IBGE, 2010) (Figura 2).

A rodovia PA-275, intercepta as rodovias PA-160 e BR-155, localiza-se na área sudeste do Estado, atendem os municípios de Eldorado dos Carajás, Curionópolis e Parauapebas. Representa o tronco viário vital para o Estado por ser a rodovia que dá acesso as principais regiões minerais do Pará. A rodovia PA-287, localiza-se na região sudeste do Estado, atende os municípios de Cumaru do Norte, Redenção e Conceição do Araguaia, chegando ao estado do Tocantins, após 
atravessar o rio Araguaia e intercepta as rodovias BR-158 e BR-155, que dão acesso os municípios de Marabá, Redenção e Santana de Araguaia na divisa com o estado do Mato Grosso (IBGE, 2010) (Figura 2).

A rodovia PA-327, intercepta na extremidade norte a PA-287 e na extremidade sul o rio Araguaia pela margem esquerda, pela margem direita do rio encontra-se com as rodovias estaduais TO-437 e TO-348 dentro do Estado do Tocantins, perpassa pelos municípios de Santana do Araguaia e Floresta do Araguaia (IBGE, 2010) (Figura 2).

No mapa de significância para a distribuição das taxas médias, padronizadas, de detecção da hanseníase na população em geral e as proporções de migração, ocorreu significância estatística para $82(56,94 \%)$ municípios do Estado do Pará, sendo 44 municípios $(53,66 \%)$ com p-valor=0,05; 22 (26,83\%) com p-valor=0,01 e 16 (19,51\%) com p-valor=0,001. A dependência espacial direta foi medida através do índice de Moran (I) com I = 0,518882. A visualização da dependência espacial através do Diagrama de Espalhamento de Moran apontou que 73 municípios apresentaram pontos de associação espacial positiva com maior concentração na área sudeste, parte da área sudoeste, norte e pequena parte do nordeste do Estado. Apresentaram pontos de associação espacial negativa apenas 09 municípios localizados na área sudeste e nordeste do Pará (Figura 3).

Figura 3 - Associação das taxas média, padronizadas, de detecção da hanseníase, na população em geral, e as proporções de migração. Distribuição espacial do Mapa de significância (a) Mapa de Moran Local (b) e Diagrama de Espalhamento de Moran (c), para os municípios do Estado do Pará, Brasil, para o período de 2006 a 2015.

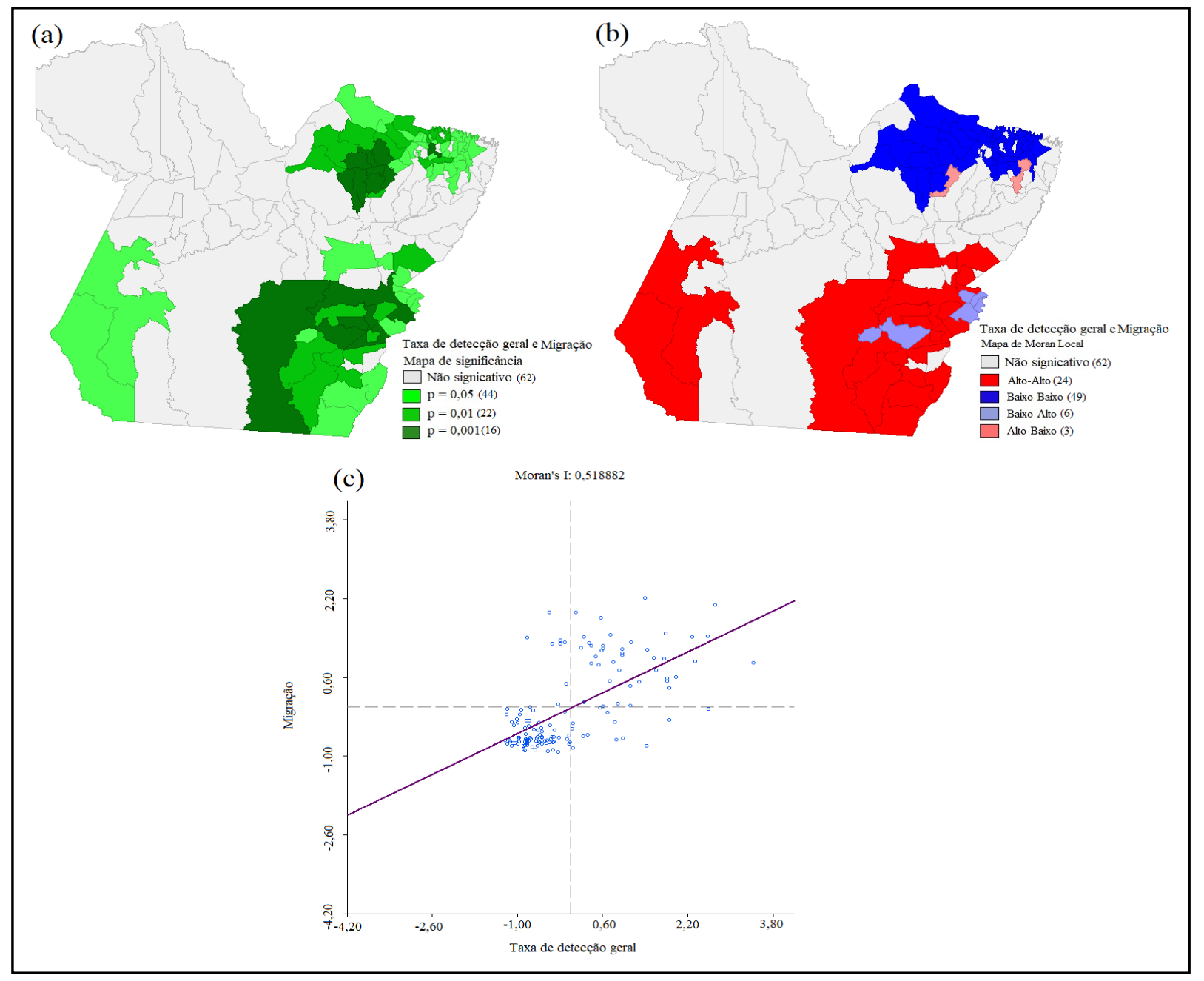

Fonte: SINAN (2017); shapefile GeoDa para Windows, versão 1.12.1.129 
No mapa de significância para a distribuição das taxas médias, padronizadas, de detecção da hanseníase em menor de 15 anos, e as proporções de migração, ocorreu significância estatística para 82 (56,94\%) municípios do Estado do Pará, sendo 44 municípios $(53,66 \%)$ com p-valor=0,05; 22 (26,83\%) com p-valor=0,01 e $16(19,51 \%)$ com p-valor=0,001. A dependência espacial direta foi medida através do índice de Moran (I) com I = 0,496093. A visualização da dependência espacial através do Diagrama de Espalhamento de Moran apontou 68 municípios com pontos de associação espacial positiva que se concentração na área sudeste, norte e pequena parte do nordeste do Estado. Municípios com pontos de associação espacial negativa foram 14 e se concentraram na área sudoeste e pequena parte na área sudeste, norte e nordeste (Figura 4).

Figura 4 - Associação das taxas média de detecção da hanseníase, em menores de 15 anos, padronizadas, e as proporções de migração. Distribuição geográfica do Mapa de significância (a), Mapa de Moran Local (b) e Diagrama de Espalhamento de Moran (c), nos municípios do Estado do Pará, Brasil, para o período de 2006 a 2015.

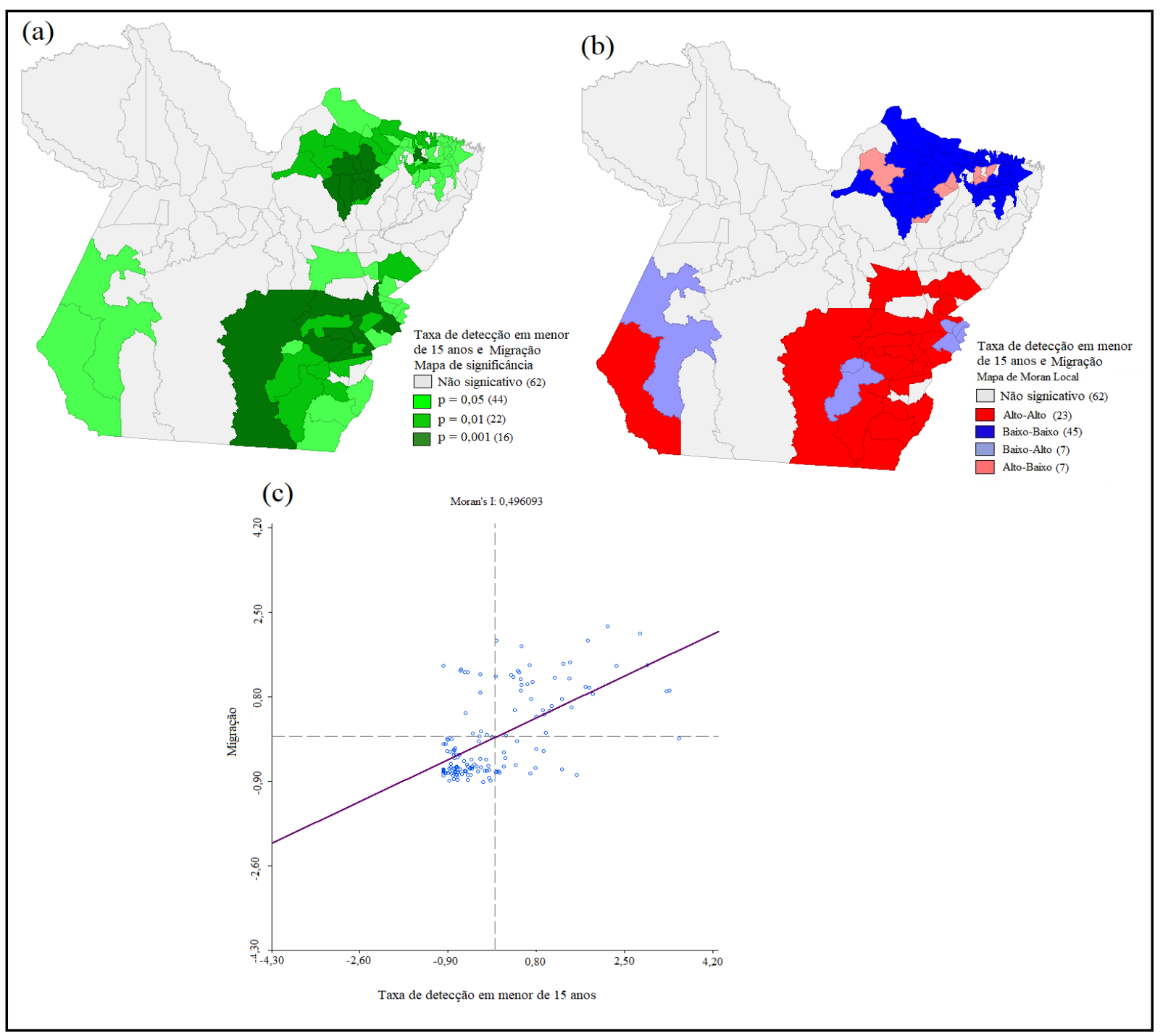

Fonte: SINAN (2017); shapefile GeoDa para Windows, versão 1.12.1.129.

Os grandes projetos de desenvolvimento econômico envolvendo mineração do solo se concentram na área sudeste do Estado, com realização de pesquisa mineral em 27 municípios, sendo que em nove deste também estão instaladas indústrias de transformação mineral e em um deles, Marabá, tem instalada uma indústria de mineração (Figura 5) (Da Silva, 2016). 
Figura 5 - Distribuição espacial dos municípios que apresentam projetos de desenvolvimento econômico, envolvendo a exploração mineral, no Estado do Pará, Brasil, em 2018.

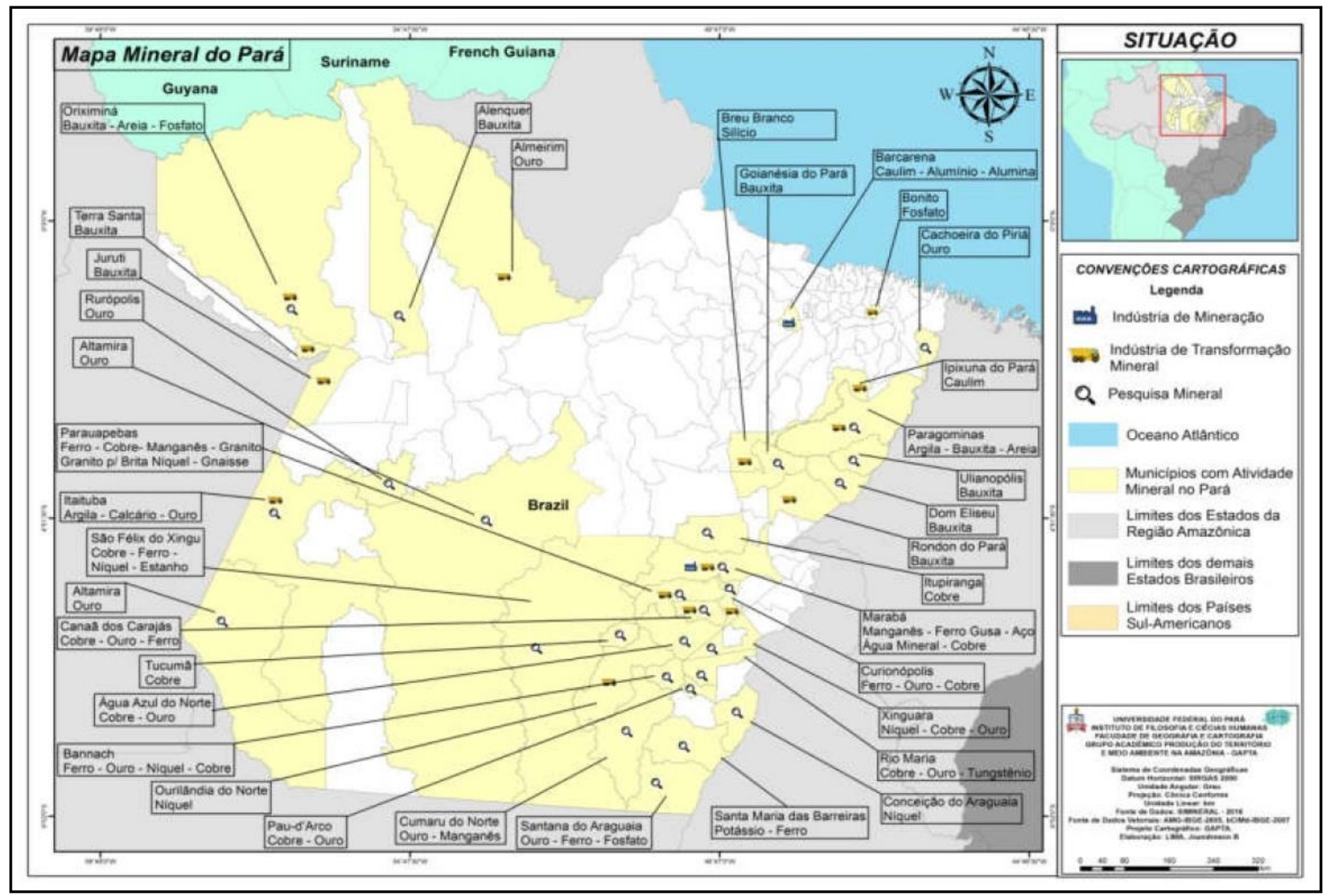

Fonte: Da Silva (2016).

\section{Discussão}

O Pará é um dos Estados que participa de todas as propostas do MS para a eliminação da hanseníase como um problema de saúde pública, entretanto este estudo ecológico revelou que as taxas de detecção de casos novos são elevadas e que a hiperendemicidade ocupa cerca de 2/3 da área geográfica do Estado.

A conformação de um corredor e de dois aglomerados (clusters) de municípios classificados como áreas de "muito hiperendemicidade" a se estender pela área sudeste do Estado, demonstram a heterogeneidade geográfica da doença e sinalizam para que medidas de promoção e prevenção da doença sejam direcionadas para essas áreas. O limite do Pará com os Estados de Tocantins, Maranhão e Mato Grosso, pela área sudeste, facilita a permanência da endemicidade considerando que esses Estados, em estudo sobre as áreas de maior risco para a transmissão da hanseníase no Brasil concentram, junto com o Pará, os clusters de mais alto risco relativo (RR maior que 3,00) para a transmissão da doença (Rodrigues et al., 2020).

Embora o Pará apresente cerca de 2/3 da área territorial classificada com hiperendêmica para hanseníase, os clusters identificados, nesta pesquisa, como área de "muito hiperendemicidade" estão localizados na área sudeste do Estado, onde se localizam os maiores projetos de reflorestamento, inclusive com presença de fábrica de MDF, que tem estimulado a formação de um polo ampliado de reflorestamento a se estender por inúmeros municípios dessa região avançando até a área nordeste do Estado (Costa et al,. 2017).

Ainda, no sudeste e sudoeste do Estado é onde se observa cerca de $87 \%$ das fazendas de criação de bovinos, que atende as produções para corte e para a produção leiteira, visando o consumo interno e a exportação. Essas áreas são favorecidas com uma rede viária composta por sete principais rodovias, quatro federais (BR-153; BR-153; BR-230 e BR-222) 
e três estaduais (PA-150; PA-279 e PA-287) que facilita o escoamento da produção agropecuária bem como o trânsito intenso de pessoas dentro do próprio Estado do Pará e entre os outros Estados vizinhos. O crescimento da pecuária e da lavoura nas áreas desmatadas de floresta densa, juntamente com a extração madeireira, dominou a economia paraense até o final da década de 1980, impondo as populações grandes custos sociais e ambientais (IBGE, 2010; Láu, 2006; Costa et al., 2017).

A autocorrelação espacial positiva das taxas de detecção de hanseníase tanto na população em geral como em menores de 15 anos de idade com a migração deixa evidente que a identificação dos três clusters localizados nas áreas sudeste, sudoeste e norte-nordeste do Estado, confirma o que já vem sendo declarado por pesquisas anteriores de que a produção do espaço temporal da doença sempre esteve ligada ao processo migratório, devido às atividades garimpeira, madeireira, agropecuária e do crescimento das periferias da área urbana dos médios e grandes municípios do estado, que pela peculiaridade da ocupação geográfica do território favorece a instalação e a manutenção de fatores de risco para a transmissão e manutenção da doença (Matos, 2005; SESPA, 2009).

A correlação das taxas de detecção da hanseníase tanto na população em geral como na de menores de 15 de idade e a proporção de migração, em transição, não se apresentou de forma polarizada, mas sim entremeada as áreas de correlação positiva, sendo maiores para a correlação entre a taxa de detecção de hanseníase em menores de 15 anos de idade e a proporção de migração, refletindo a magnitude e a tendência da doença assim como a força de transmissão recente da endemia (Anselin, 1995; Souza \& Rodrigues, 2015).

Os diferentes investimentos sobre pesquisas minerais, instalação de indústria de transformação mineral e de indústria de mineração, tornaram o Estado do Pará uma área de forte crescimento econômico do país, principalmente após a descoberta das jazidas de ouro em Serra Pelada, na década de 1980, e das jazidas de ferro localizadas no município de Carajás. Essa área de exploração é sustentada pelo Plano Nacional da Mineração previsto para execução até 2030. Até o ano de 2016, os investimentos na busca por minerais estavam situados em 25\% (36/144) dos municípios do estado, sendo que destes, 52,78\% (19/36) situavam-se na área sudeste do Pará (Da Silva, 2016).

A ocorrência da hanseníase na Amazônia Legal tem sido estudada como associada ao modo de ocupação do território, que inicialmente foi fomentada por Políticas de crédito a partir de 1970, que provocou uma demanda crescente de pessoas à Região que nem sempre contavam com infraestrutura adequada para a recepção dos migrantes, o que contribuía para a precária condição de vida. Atualmente, essa área do estado continua a apresentar fluxo migratório fomentado pelo desenvolvimento dos grandes projetos de mineração que tem atraído trabalhadores e familiares em busca de melhor qualidade de vida (Silva et al., 2010).

A migração explica, portanto, na instalação da hanseníase no Estado e na sua manutenção em regiões distintas, entretanto a persistência em áreas específicas pode estar associada a outros fatores, como uma maior vulnerabilidade ambiental ou mesmo individual e baixos investimentos de ampliação das atividades de Atenção Primária da Saúde (APS), através da expansão oportuna da cobertura populacional da Estratégia Saúde da Família (ESF) (Alencar et al., 2012; Freitas et al., 2014; WHO, 2016). 


\section{Conclusão}

O estudo da autocorrelação espacial da hanseníase com a migração, utilizando técnicas de análise espacial, mostrou ser eficaz e de grande valia para a compreensão da situação epidemiológica da hanseníase no estado do Pará. Os clusters identificados revelaram que a distribuição da doença não é homogênea e que o impacto desta atinge áreas de maior produção econômica do Estado. Essas áreas devem ser consideradas como prioritárias para as intervenções, planejadas estrategicamente, para o combate do problema. Esta pesquisa identifica, também, a importância de que a sustentação da endemicidade no estado do Pará, requisita mais pesquisas que utilizem distintos questionamentos para aprimorar a compreensão de outros fatores que condicionam, ou mesmo determinam, a manutenção da doença em cerca de 2/3 da área territorial do Estado do Pará. O quadro epidemiológico observado sugere pesquisas com novas abordagens metodológicas para a compreensão dos fatores que condicionam a manutenção de altas taxas de detecção no Pará.

\section{Referências}

Alencar, C. H. M., Ramos Júnior, N. A., Dos Santos, E. S., \& Richter, J. (2012). Clusters of leprosy transmission and of late diagnosis in a highly endemic area in Brazil: focus on different spatial analysis approaches. Trop Med Int Health. 17, 518-25

Anselin, L. (2016). GeoDaTM software for geodata analysis: version GeoDa versão: 1.12.1.129. The Center for Spatial data Science. https://geodacenter.github.io/workbook/1_datascience/lab1.html.".

Anselin, L. (1995). Local Indicators of Spatial Association - LISA Geographical Analysis, 27(2), 93/115

Brasil. Ministério da Saúde. Secretaria de Vigilância Epidemiológica. (2014a). Guia de Vigilância em Saúde. Caderno Hanseníase. Versão eletrônica, Brasília: MS, 2014. 345 - 377.

Brasil. Ministério da Saúde. Secretaria de Vigilância Epidemiológica em Saúde. (2014b). Situação Epidemiológica da Hanseníase no Brasil. <http://portalsaude.saude.gov.br/index.php?option=com_content\&view=article\&id=10995 \&Itemid=661>

Brasil. Ministério da Saúde. Secretaria de Vigilância em Saúde. Departamento de Vigilância das Doenças Transmissíveis. (2016). Diretrizes para vigilância, atenção e eliminação da Hanseníase como problema de saúde pública: manual técnico-operacional [recurso eletrônico] / Ministério da Saúde, Secretaria de Vigilância em Saúde, Departamento de Vigilância das Doenças Transmissíveis. - Brasília: Ministério da Saúde, 58p

Brook, C. E., Beauclair, R., Ngwenya, O., Worden, L., Ndeffo-mbah, M., Lietman, T. M., Satpathy, S. K., Galvani, A. P., \& Porco, T. C. (2015). Spatial heterogeneity in projected leprosy trends in India. Parasit Vectors 2015; 8(1), 542

Costa, E. J. M. (2011). Planejamento Territorial, Gestão de Políticas Públicas e Descentralização Regional: a experiência do Estado do Pará. DRd. 1(1)

Cury, M. R. C. O., Paschoal, V. D. A., Nardi, S. M., Chierotti, A. P., Rodrigues Júnior, A. L., \& Chiaravalloti-Neto, F. (2012). Spatial analysis of leprosy incidence and associated socioeconomic factors. Rev Saúde Pública. 46 (1), 110-8

Da Silva, J. M. (2016). Dinâmica territorial da mineração na Mesorregião Sudeste do estado Pará - Região Norte do Brasil. Programa de Pós-Graduação em Geografia. Universidade Federal do Pará. https://www.researchgate.net/publication/311323973.

Costa, M. R. T. R., Homma, A. K. O., Rebello, F. K., Souza Filho, A. P.S., Fernandes, G. L. C., \& Baleixe, W. (2017). Atividade agropecuária no Estado do Pará-Belém, PA. Embrapa Amazônia Oriental, 2017. 174 (Documentos / Embrapa Amazônia Oriental, ISSN 1983-0513; 432)

Fundação Amazônia de Amparo a Estudos e Pesquisas do Pará (FAPESPA). (2016). Diagnóstico Socioeconômico e Ambiental do Estado do Pará e Regiões de Integração. Apresentação proferida por Eduardo Costa, Diretor Presidente da FAPESPA. www.fapespa.pa.gov.br.

Freitas, L. R.; Duarte, E. C.; \& Garcia, L. P. (2014). Leprosy in Brazil and its association with characteristics of municipalities: ecological study, 2009-2011. Trop Med Int Health. 19 (10), 1216 - 1225

Giraldin, R. M. (2015). Entre trânsitos e consumos: a presença dos trabalhadores de Belo Monte em Altmira, PA. Dissertação de mestrado apresentada ao Programa de Pós - Graduação em Antropologia Social, Departamento de Antropologia (DAN), Universidade de Brasília (UnB)

Instituto Brasileiro de Geografia e Estatística (IBGE). (2010) Censo demográfico 2010. http:/www.ibge.gov.br/home/estatística/censo 2010.

Instituto de Pesquisa Econômica Aplicada (IPEA). (2013). Programa das Nações Unidas para o Desenvolvimento (PNUD). Fundação João Pinheiro (FJP). (2013). Atlas do Desenvolvimento Humano: http://www.atlasbrasil.org.br/2013/.

Láu, H.D. (2006). Pecuária no Estado do Pará: índices, limitações e potencialidades. Belém, PA. Embrapa Amazônia Oriental. 36p (Embrapa Amazônia Oriental. Documentos, 269)

Matos, R. (2005). Periferias de grandes cidades e movimentos populacionais. Cadernos Metrópole. 13: 71-105

Monken, M. \& Barcellos, C. (2005). Vigilância em saúde e território utilizado: possibilidades teóricas e metodológicas. Cad. Saúde Pública, Rio de Janeiro, 21(3), 898-906. 
Research, Society and Development, v. 10, n. 1, e1810111164, 2021

(CC BY 4.0) | ISSN 2525-3409 | DOI: http://dx.doi.org/10.33448/rsd-v10i1.11164

Monteiro, L. D., Martins-Melo, F. R. Brito, A. L., Alencar, C. H., \& Heukelbach, J. (2015). Padrões espaciais da hanseníase em um estado hiperendêmico no Norte do Brasil, 2001 - 2012. Rev. Saúde Pública. 49, 84

Nunes, F. G. (2013). Análise Exploratória Espacial de indicadores de desenvolvimento Socioambiental das Regiões de Planejamento do Norte e Nordeste Goiano. Ateliê Geográfico. 7 (1): 237-259

Pereira, A. S., et al. (2018). Metodologia da pesquisa científica. [e-book]. Santa Maria. Ed. UAB/NTE/UFSM. https://repositorio.ufsm.br/bitstream/handle/1/15824/Lic_Co mputacao_Metodologia-Pesquisa-Cientifica.pdf?sequence=1

Rodrigues, R. N., Leano, H. A. M., Bueno, I. C., Araújo, K. M. F. A., \& Lana, F. C. F. (2020). Áreas de alto risco de hanseníase no Brasil, período 2001-2015. Rev Bras Enferm. 2020;73(3):e20180583

Secretaria de Estado de Saúde Pública do Pará (SESPA). (2009). Hanseníase no Pará: fatores que interferem na manutenção dos elevados coeficientes de deteç̧ão. Relatório de Pesquisa Operacional. https://english.aifo.it/research/leprosy/pesquisa_hanseniasi_para.pdf.

Silva, D. R. X., Ignoti, E., Souza-Santos, R. Hacon, S. S. (2010). Hanseníase, condições sociais e desmatamento na Amazônia brasileira. 2010. Rev Panam Salud Publica; 27 (4), 268-75

Souza, C. D. F, \& Rodrigues, M. (2015). Magnitude, Tendência e Espacialização da hanseníase em menores de 15 anos no estado da Bahia, com enfoque em áreas de risco: um estudo ecológico. Hygeia 11 (20), 201 - 212

World Health Organization (WHO). (2015). Global leprosy update, time for action, accountability and inclusion. Wkly Epidemiol Rec. 2016. 91(35), 405-20

World Health Organization (WHO). (2016). Weekly Epidemiol Rec. 35 (91), 405 - 420. http://www.w ho.int/wer. 